\title{
HPV Vaccine Hesitancy Among Medical Students in China: A Multicenter Survey
}

\author{
Liangru Zhou, Jian Wang, Pengxin Cheng, Yue Li, Guoxiang Liu* and Xin Zhang* \\ School of Health Management, Harbin Medical University, Harbin, China
}

Human papillomavirus (HPV) is the most common genital tract virus infection and can cause genital warts and cervical cancer. This multicenter study examined HPV information sources, vaccine hesitancy, and the association between the two variables.

OPEN ACCESS

Edited by:

Bridget Kelly,

RTI International, United States

Reviewed by:

Rosalia Maria Ragusa

University Hospital Polyclinic Vittorio

Emanuele, Italy

Francesco Paolo Bianchi,

University of Bari Aldo Moro, Italy

Nora B. Henrikson

Kaiser Permanente Washington Health

Research Institute, United States

*Correspondence:

Guoxiang Liu

Igx6301@163.com

Xin Zhang

zhangxinzhx0801@126.com

Specialty section:

This article was submitted to

Public Health Education and

Promotion,

a section of the journal

Frontiers in Public Health

Received: 13 September 2021

Accepted: 11 January 2022

Published: 21 February 2022

Citation:

Zhou L, Wang J, Cheng P, Li Y, Liu G and Zhang $X$ (2022) HPV Vaccine Hesitancy Among Medical Students in

China: A Multicenter Survey.

Front. Public Health 10:774767.

doi: 10.3389/fpubh.2022.774767
An online survey of HPV information sources and vaccine hesitancy was conducted among Chinese medical students. The World Health Organization (WHO) Vaccine Hesitancy 3Cs model was used to evaluate reasons for respondents' vaccine hesitancy. A probit model was used to investigate the association between vaccine information sources and vaccine hesitancy. The reported rate of vaccine hesitancy was $62.36 \%$. Convenience was the primary factor for vaccine hesitancy in medical students, and 19\% used a single source to obtain vaccine information. A multivariate analysis revealed that master degree and above were 33\% less likely to be hesitant about the HPV vaccine than first grade students. Respondents receiving HPV information through doctor were $8 \%$ less likely to report vaccine hesitancy than those receiving information from other information channels. HPV vaccine hesitancy requires more attention. Future studies could examine whether increasing vaccination locations and dissemination of information about the safety and effectiveness of HPV vaccines as well as using Internet media would help reduce medical students' vaccine hesitancy and expand HPV vaccine coverage.

Keywords: HPV vaccine, vaccination information, hesitancy, 3Cs model, medical students

\section{INTRODUCTION}

Human papillomavirus (HPV) is the most common genital tract infection virus. Most sexually active women and men will be infected with the virus at some point in their lives, some repeatedly (1). HPV plays a role in the development of skin diseases such as head and neck cancer, cervical cancer, male genital cancer, and condyloma acuminatum. The HPV subtypes are classified into high-risk (HPVl6, 18, 31, 33, 35, 45, etc.) and low-risk types (HPV6, 11, 30, 39, 42, 43, etc.) $(2,3)$. Almost all cases of cervical cancer (99\%) are associated with high-risk HPV infection (4).

According to the GLOBOCAN 2020 reported that there were 604,000 new cases of cervical cancer and 342,000 deaths worldwide in 2020; 110 thousand new cases of cervical cancer and 59,000 deaths were reported in China, making it the second-largest burden of cervical cancer in the world (5). The prevalence of HPV-related head and neck cancer, especially throat cancer, is increasing rapidly. It is expected that the number of patients with HPV-positive throat cancer will exceed that of patients with cervical cancer in the next 15-20 years (6). 
Prophylactic HPV vaccine is an effective way to prevent HPV-related diseases, including cervical cancer (7). The incidence and costs of cervical cancer already significantly decreasing in Italy (8). And vaccination does not require any major lifestyle changes (9).

HPV vaccination has been implemented on a large scale and achieved remarkable results since the vaccine's production and marketing in 2006 (10). Studies in several countries and regions have confirmed that HPV vaccination is cost-effective (11-14).

However, the emergence of vaccine hesitancy has dampened public enthusiasm for vaccination. In 2019, the WHO listed vaccine hesitancy as one of the top 10 threats to global health (15). Vaccine hesitancy refers to either reluctance to accept or rejection of vaccination when it is available (16). A decline in the HPV vaccination rate due to $H P V$ vaccine hesitancy has been reported in many countries and regions. For example, in 2013, after the media reported that vaccination with untested vaccines would cause adverse events, HPV vaccine hesitancy spread widely in Japan, resulting in a significant decline in the Japanese vaccination rate (17). A study of Brazilian medical students suggested that vaccine hesitancy is the fundamental determinant of the low HPV vaccination rate (18). Although studies have been conducted on HPV infection awareness and knowledge in China $(19,20)$, evidence concerning Chinese HPV vaccine hesitancy is lacking. According to the $\mathrm{WHO}$, confidence is one of the three major determinants of vaccine hesitancy (21). Undoubtedly, unproven negative news has undermined public trust in HPV vaccines. However, for the public, it is challenging to determine authentic and valid facts in the vast pool of available information. Transparent and accurate information is essential for rebuilding vaccine confidence (22). In addition, it is necessary to understand the public's sources of information and improve the efficiency of information transmission.

The HPV vaccine entered the Chinese market as a selffunded vaccine in 2016, with domestic approval of bivalent $\mathrm{HPV}$ vaccines for women aged 9-45; quadrivalent $\mathrm{HPV}$ vaccines for women aged 20-45; and 9-valent HPV vaccines for women aged 16-26 (23). In November 2020, mainland China reduced the minimum age for quadrivalent vaccines to 9 years. China's HPV vaccine has been on the market for only a few years compared with other countries, and some people adopt the "wait-and-see" approach regarding vaccines, resulting in low vaccination rates. Many medical students are also target populations for the HPV vaccine. In addition, as the future generations of medical personnel, they bear the responsibility of promoting the health of the entire population and play an important role in the promotion and popularization of $\mathrm{HPV}$ vaccination. To investigate the factors related to HPV vaccine hesitancy among medical students and estimate the association between the source of information and vaccine hesitancy, the author developed an information source and hesitancy questionnaire and conducted a questionnaire survey with Chinese medical students.

\section{MATERIALS AND METHODS Study Design and Implementation}

At the beginning of November 2020, a preliminary survey was conducted at the author's medical university. The purpose was to improve the preset items and test the practicality of the survey, including whether the question expressions were easy to understand, and to improve the questionnaire through information from pre-survey feedback. The formal survey was conducted from mid-January to the end of March, and a crosssectional multicenter survey on HPV information sources and hesitancy was conducted for medical students at the school. The investigation was conducted anonymously. Participants were recruited at Harbin Medical University, Hebei Medical University and Chengdu University of Traditional Chinese Medicine. Participants must be students of Chinese nationality. At the beginning of the survey, the participants were asked whether they would voluntarily participate in the research, and indicated that participants are free to withdraw from the research. The questionnaire takes 3-5 min to fill out. Each respondent received $2 \mathrm{RMB}$ in cash as remuneration, which was paid through online payment. The study was open to people of any sex. To maintain survey quality, we implemented the following controls: (1) All questions were set as compulsory to avoid missing responses; (2) When a respondent chose the same option for all questions, they were prompted to repeat their answers, and the survey would not proceed until they had done so.

\section{Data Collection and Questionnaire Measures}

A total of 850 medical students were invited. A questionnaire for medical students was compiled, and it included four parts. The first part of the questionnaire reported the purpose and objectives of the research, asked the participants whether they would voluntarily participate in the study, and indicated that they were free to withdraw from the research. The second part of the questionnaire surveyed participants' demographic information, sex, age, grade, type of family residence, partner status, health behavior, whether they had internship experience in a medical institution, and monthly consumption, which was food and shopping expenditures, excluding large expenditures like expensive electronic products.

The third part investigated participants' vaccine hesitancy and their reasons. Vaccine hesitancy refers to delay in acceptance or refusal of vaccines despite availability of vaccine services. Hesitancy was scored as the percentage of participants endorsing each item. The WHO Vaccine Hesitancy 3Cs model classifies the reasons for vaccine hesitancy as complacency, convenience, and confidence. Complacency refers to the underestimation of the harm the disease can cause and doubts about the necessity of vaccine use. Confidence refers to the trust in the safety, effectiveness, health system, and administrators of vaccines. Convenience refers to vaccine supply capacity, vaccine price acceptance, and availability of vaccination services. Following this classification, and in accordance with China's social and 
TABLE 1 | Participants' characteristics and vaccine hesitancy.

\begin{tabular}{|c|c|c|c|}
\hline Characteristics & $\begin{array}{c}\text { Sample } \\
N(\%)\end{array}$ & $\begin{array}{c}\text { Hesitancy } \\
\quad N(\%)\end{array}$ & $P$-value \\
\hline Total & $728(-)$ & $454(-)$ & - \\
\hline Sex & & & 0.00 \\
\hline Female & $450(62)$ & $258(57)$ & \\
\hline Male & $278(38)$ & $196(43)$ & \\
\hline Age & & & 0.07 \\
\hline $16-26$ & $538(74)$ & $325(72)$ & \\
\hline $27-45$ & $190(26)$ & $129(28)$ & \\
\hline Grade & & & 0.00 \\
\hline First year of university & $35(5)$ & $31(7)$ & \\
\hline The second year of university & $116(16)$ & $91(20)$ & \\
\hline The third year of university & $104(14)$ & $84(19)$ & \\
\hline Fourth grade of university & $65(9)$ & $37(8)$ & \\
\hline Fifth grade of university & $27(4)$ & $15(3)$ & \\
\hline Master degree and above & $381(52)$ & $196(43)$ & \\
\hline Type of residence & & & 0.80 \\
\hline Urban & $565(78)$ & $351(77)$ & \\
\hline Rural & $163(22)$ & $103(23)$ & \\
\hline Partner & & & 0.00 \\
\hline No partner & $629(86)$ & $376(83)$ & \\
\hline Have a partner & $99(14)$ & $78(17)$ & \\
\hline Hospital Internship & & & 0.42 \\
\hline Yes & $621(85)$ & $391(86)$ & \\
\hline No & $107(15)$ & $63(14)$ & \\
\hline Monthly consumption (monthly) & & & 0.06 \\
\hline$\leq \$ 300$ & $427(41)$ & $254(56)$ & \\
\hline$>\$ 300$ & 301 (59) & $200(44)$ & \\
\hline Hours of exercise (weekly) & & & 0.01 \\
\hline$\leq 1 \mathrm{~h}$ & $277(38)$ & $159(35)$ & \\
\hline $1 \leq 3 h$ & $341(47)$ & $232(51)$ & \\
\hline$>3 \mathrm{~h}$ & $110(15)$ & $63(14)$ & \\
\hline
\end{tabular}

cultural environment, 13 possible reasons were included for participants to choose from, and open items were included for participants to add additional reasons.

The fourth part investigated HPV and vaccine information sources. Sources include online media, traditional media represented by newspaper, television and radio, offline publicity such as school lectures and subway advertisements and personto-person communication among doctors, family, and friends. The number of channels was collected from the results of the information sources and counted according to the reported information sources. The range of the number of channels was $0-8$.

\section{Statistical Analyses}

Statistical analysis was performed using Stata 15.0. Categorical variables were analyzed using chi-squared tests, and continuous variables were analyzed using one-way analysis of variance. A probit model was used to identify whether the information source and number of information sources were the key factors for
TABLE 2 | Reasons for respondents' vaccine hesitancy.

\begin{tabular}{lc}
\hline Reasons & Yes, $\boldsymbol{N}$ (\%) \\
\hline Complacency & \\
No vaccination was considered necessary & $190(42)$ \\
Convenience & \\
Don't know where to get vaccinated & $264(58)$ \\
The vaccination site is far away and requires a long journey & $270(60)$ \\
Repeated vaccination doses, feeling troublesome & $274(60)$ \\
Don't know where to get good/reliable information & $287(63)$ \\
& Confidence \\
Hear or read negative news & $230(51)$ \\
Fear of needles & $187(41)$ \\
Someone told me they had a bad reaction & $233(51)$ \\
Had a bad experience with previous vaccinations due to health & $185(41)$ \\
clinics/vaccinators & \\
Worried about vaccine safety/side effects & $280(62)$ \\
I've been told that vaccines aren't safe & $188(41)$ \\
Vaccination had bad physiological and psychological reactions & $190(42)$ \\
before &
\end{tabular}

hesitancy, and the marginal utility of the regression coefficients of the probit model was calculated. In the analysis, we controlled for demographic characteristics of the respondents, including age, sex, place of residence (rural or urban), monthly living expenses, and weekly exercise duration. A $P<0.05$ was considered statistically significant throughout the study.

\section{Ethics Approval and Consent to Participate}

The study was approved by the Medical Ethics Committee of Harbin Medical University (HMUIRB20210006). Respondents were informed that they were free to withdraw from the study and that all data would be strictly confidential and used only for scientific analysis.

\section{RESULTS}

\section{Participants' Characteristics}

A total of 728 respondents responded to the questionnaire. Table 1 shows participants' baseline characteristics. Among the participants, $62 \%$ were women, and $38 \%$ were men. All were between 16 and 45 years old. A total of $52 \%$ of the respondents are masters and above. A total of $78 \%$ of respondents lived in urban areas, and $86 \%$ did not have a partner. Overall, $85 \%$ of them had hospital internship experience, $41 \%$ had a monthly consumption of $<\$ 300$, and $38 \%$ exercised $<1$ h per week.

\section{Vaccine Hesitancy and Reasons for Vaccine Hesitancy}

Among the respondents, 62.36\% (454/728) reported having vaccine hesitancy. Participants of different sexes $(P=0.00)$, different grades $(P=0.00)$, with or without partners $(P=0.00)$, and doing different amounts of exercise $(P=0.01)$ reported different levels of vaccine hesitancy (Table 1). 


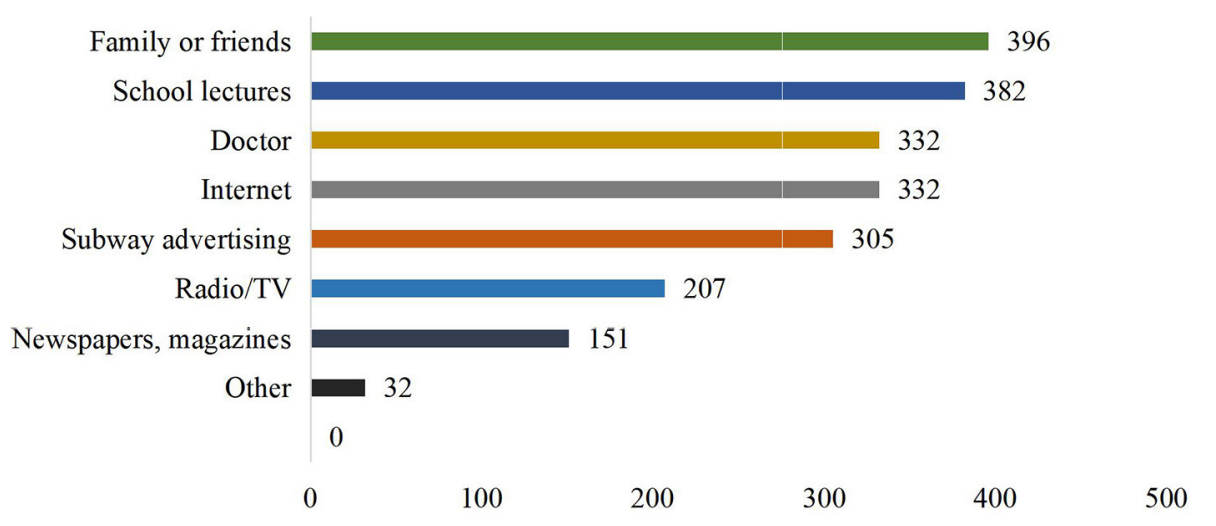

FIGURE 1 | Distribution of respondents' information sources (sorted).

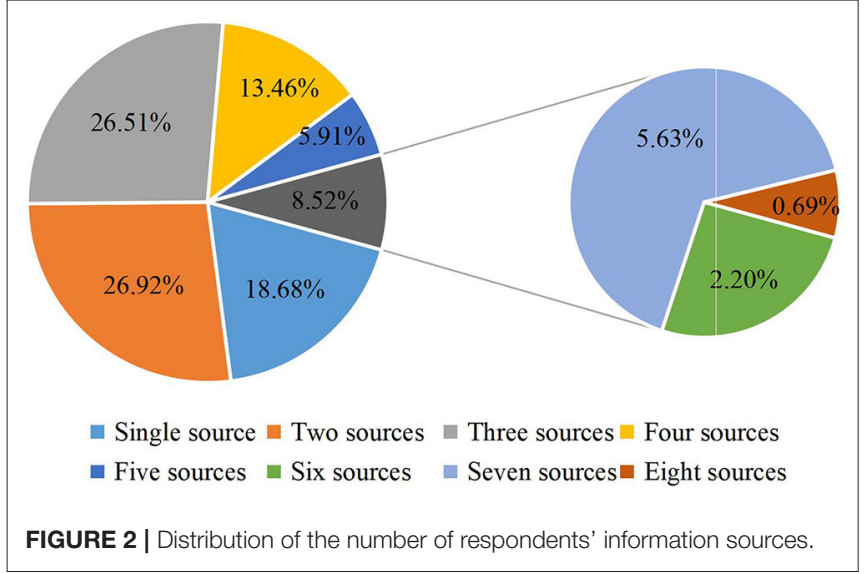

The reasons for respondents' vaccine hesitancy are shown in Table 2. Overall, $42 \%$ of respondents believed that HPV vaccination was unnecessary. Among the convenience items, $63 \%$ of participants did not know where to obtain reliable information. Regarding the confidence items, most respondents worried about vaccine safety or side effects (62\%), 51\% reported negative reactions of others, and 51\% reported that they had heard or read negative news.

\section{HPV Information Sources}

The main source of HPV knowledge was family or friends members, school lectures and doctors, followed by the internet, subway advertising and radio/television (Figure 1). Regarding the number of information channels, respondents with two sources $(26.92 \%)$ and three sources $(26.51 \%)$ accounted for the majority (Figure 2).

\section{Multivariate Analysis}

The results of the probit regression model showed that grade and doctor information source were statistically significant at the 0.05 level. The higher the grade, the lower the possibility of vaccine hesitancy. People who obtained information from the doctor had a reduced probability of vaccine hesitancy compared to other sources (Table 3). The results of marginal utility showed that the average partial effect for grade was -0.33 , indicating that HPV vaccine hesitancy among master degree and above respondents was $33.00 \%$ lower than that among first grade. The average partial effect of not having a partner was -0.11 , indicating that the probability of HPV vaccine hesitancy for those without a partner was $11 \%$ lower than for those with a partner, even if it is not statistically significant. The average partial effect of doctor sources of information was -0.08 , indicating that respondents receiving HPV information through the Internet were $8 \%$ less likely to report vaccine hesitancy than those receiving information from other information channels.

\section{DISCUSSION}

Our study targeted Chinese medical students and examined the incidence and reported reasons for HPV vaccine hesitancy among Chinese medical students. It also investigated the sources and number of sources of HPV vaccination information for medical students and explored the correlation between vaccination information and vaccine hesitancy.

The study found a high degree of HPV vaccine hesitancy among the people surveyed, with more than $60 \%$ of the respondents reporting hesitancy about the HPV vaccine. The findings showed that some people do not see the necessity of vaccination. This may reflect a cognitive bias, as an optimistic bias, namely, that they are unlikely to develop cervical cancer, or discounting the future, that is, lack of concern about their middle or old age in the future. Beyond this, HPV infection might be stigmatized by some as related to life misconduct. Some respondents may believe that they could avoid contracting the virus by having the same sexual partner. However, global observations suggest that $75 \%$ of sexually active adults develop HPV at some point in their lifetime (24).

Convenience was reported as an important factor in the respondents' hesitancy about the HPV vaccine. Participants reported issues with not knowing where to get reliable vaccination information (63\%) and not knowing where to 
TABLE 3 | Determinants of vaccine hesitancy using Probit model.

\begin{tabular}{|c|c|c|c|c|c|c|}
\hline Hesitancy & Coef. & Std. Err. & $\mathbf{z}$ & $\mathbf{P}>\mathbf{z}$ & $95 \% \mathrm{Cl}$ & Margins \\
\hline Years of age, 16-26 & -0.07 & 0.12 & -0.53 & 0.60 & $(-0.31,0.18)$ & -0.02 \\
\hline Male & 0.12 & 0.11 & 1.06 & 0.29 & $(-0.1,0.34)$ & 0.04 \\
\hline \multicolumn{7}{|l|}{ Grade } \\
\hline The second year of university & -0.38 & 0.32 & -1.17 & 0.24 & $(-1.01,0.25)$ & -0.10 \\
\hline The third year of university & -0.30 & 0.33 & -0.92 & 0.36 & $(-0.95,0.34)$ & -0.07 \\
\hline Fourth grade of university & -0.92 & 0.34 & -2.73 & 0.01 & $(-1.58,-0.26)$ & -0.28 \\
\hline Fifth grade of university & -0.93 & 0.39 & -2.42 & 0.02 & $(-1.69,-0.18)$ & -0.29 \\
\hline Master degree and above & -1.05 & 0.31 & -3.39 & 0.00 & $(-1.66,-0.44)$ & -0.33 \\
\hline Urban & -0.11 & 0.12 & -0.90 & 0.37 & $(-0.35,0.13)$ & -0.04 \\
\hline No partner & -0.31 & 0.17 & -1.85 & 0.07 & $(-0.64,0.02)$ & -0.11 \\
\hline$\leq \$ 300$ & -0.04 & 0.11 & -0.40 & 0.69 & $(-0.26,0.17)$ & -0.02 \\
\hline Internship & 0.02 & 0.14 & 0.13 & 0.89 & $(-0.26,0.3)$ & 0.01 \\
\hline \multicolumn{7}{|l|}{ Hours of exercise (weekly) } \\
\hline $1 \leq 3 h$ & -0.03 & 0.12 & -0.27 & 0.79 & $(-0.26,0.2)$ & -0.01 \\
\hline$>3 h$ & -0.18 & 0.15 & -1.21 & 0.23 & $(-0.48,0.11)$ & -0.06 \\
\hline \multicolumn{7}{|l|}{ Information sources } \\
\hline Family or friends & 0.12 & 0.10 & 1.22 & 0.22 & $(-0.07,0.32)$ & 0.04 \\
\hline Doctor & -0.23 & 0.11 & -2.13 & 0.03 & $(-0.44,-0.02)$ & -0.08 \\
\hline Internet & -0.11 & 0.12 & -0.95 & 0.34 & $(-0.35,0.12)$ & -0.04 \\
\hline Radio/TV & -0.12 & 0.13 & -0.93 & 0.35 & $(-0.36,0.13)$ & -0.04 \\
\hline School lectures & 0.02 & 0.11 & 0.21 & 0.83 & $(-0.19,0.23)$ & 0.01 \\
\hline Public welfare publicity and subway advertising & 0.14 & 0.11 & 1.27 & 0.20 & $(-0.08,0.36)$ & 0.05 \\
\hline Newspapers, magazines & -0.03 & 0.14 & -0.24 & 0.81 & $(-0.31,0.24)$ & -0.01 \\
\hline Other & -0.35 & 0.23 & -1.48 & 0.14 & $(-0.81,0.11)$ & -0.12 \\
\hline _cons & 1.62 & 0.39 & 4.15 & 0.00 & $(0.85,2.38)$ & - \\
\hline
\end{tabular}

Cl, confidence interval.

receive vaccination (58\%). Medical students in Chongqing, China, have reported information as an important obstacle to receiving HPV vaccination (25). A total of $60 \%$ of participants reported hesitancy on the grounds of repeated vaccination doses and inconvenient vaccination. The percentage of participants who reported having to travel long distances was $60 \%$. This may be because HPV vaccination has clear time intervals and dosage requirements. This partly increases the traffic cost of vaccination. To shorten the time cost and improve the accessibility of vaccination, vaccination sites could be added (26) by using mobile vaccination vehicles to provide vaccination services or by providing vaccination services in schools or pharmacies. Although this would improve the availability of vaccination, ensuring vaccine circulation and meeting storage standards should not be ignored; these need joint participation by the regulatory authorities. Opening online or telephone appointments is another way to make vaccination easier. SMS reminder services could also improve the rate of full vaccination.

The safety and effectiveness of vaccines was another key factor reported by medical students in vaccine hesitancy (62\%). Research on the South East Asia and Western Pacific Regions has reported that safety concerns are related to vaccine hesitancy (27). Safety concerns are also related to insufficient knowledge about HPV. In fact, the safety and effectiveness of the HPV vaccine have been widely demonstrated. The Global Advisory
Committee on Vaccine Safety of the WHO evaluates HPV vaccine performance as "extremely safe" (2).

The US Centers for Disease Control and Prevention noted that many people who get the HPV vaccine experience no side effects. The most common side effects are usually mild. Severe allergic reactions following vaccination are rare (28). Providing scientific information to the public is an indispensable way to reduce the impact of the vaccine controversy (29). Fear of needles $(41 \%)$ is also a factor in vaccine hesitancy. Taddio's study confirmed that fear of pain is one of the major barriers to vaccination (30). In future vaccine development, the vaccine dosage type could reduce the fear of needles.

The main sources of HPV vaccine knowledge for medical students are friends/family, school lectures, doctors, and the Internet. Fewer people report newspapers, radio/television sources. A study on HPV in Italy also pointed out that fewer and fewer people get vaccination information through TV, radio or newspapers (31). Medical students who rely on family and friends for vaccine information are more likely to report vaccine hesitancy, although this result is not significant $(P>$ 0.05). As parents and friends are the main source of social communication among medical students, they can always get information from them. This experience also includes views and attitudes toward the HPV vaccine. This means that, 
in addition to providing more vaccine information for the respondents, the popularization of the HPV vaccine information among their parents or friends may also indirectly increase respondents' knowledge of the HPV vaccine. Respondents who received information from doctors were less likely to report vaccine hesitancy $(P<0.05)$. As a professional source of information, doctors can provide authoritative HPV vaccine information to dispel people's doubts about the vaccine. A study in Florida has noted that doctors' advice on the HPV vaccine predicts vaccination status (32). Therefore, training healthcare providers to address common vaccine problems (33) may be a feasible way to reduce vaccine hesitancy. On the one hand, public health providers can provide those considering vaccinations with any information they may need (34). People who obtain information from Internet sources are less likely to report vaccine hesitancy. Young students are used to finding solutions online when experiencing problems. The Internet plays an important role in disseminating information about vaccination (35). Using the Internet as a key carrier of vaccine information may effectively increase respondents' awareness of the HPV vaccine. However, few studies also suggest that social media platforms have become a common source of vaccine information and false information (36). To reduce the spread of false information, it is necessary to increase the publication of accurate vaccine information and the supervision of online information.

HPV vaccine hesitancy needs to be given more attention, especially among medical students and medical staff. These people are not only HPV vaccine recipients, but also a key force in promoting HPV vaccine coverage. The widespread occurrence of HPV vaccine hesitancy will affect vulnerable populations including newborns and pregnant women. This study shows that the uncertainty of the safety of HPV vaccines is the reason why medical students hesitate to produce vaccines. Concerns about vaccine safety have also appeared in the hesitancy of COVID-19 vaccine studies in the United States and Canada $(37,38)$.

It is necessary to carry out health education activities on the safety and effectiveness of vaccines. This may be an effective way to reduce the misunderstanding of HPV vaccines by medical students and the public and promote uptake of HPV vaccines. The target population of health education can be parents, teachers, public health service providers, specialist doctors and vaccinators themselves, and these people can also become implementers of health education. Strategies are as follows: (1) Providing HPV vaccine information. When the vaccination audience is vaccinated with other vaccines, vaccination service providers take the initiative to provide information about HPV vaccines (39). Distributing vaccine brochures at vaccination sites, posting vaccine posters, providing vaccine appointment explanation services, etc.; (2) Health care providers conduct health lectures in schools to explain the knowledge and preventive measures of cervical cancer and genital diseases; (3) Doctors can also promote correct information to the right-age vaccinated population and their parents, and encourage this population to be vaccinated. (4) Health professionals or young doctors have often been interviewed to get quick and easy guidance on procedures or processes that could be transferred to the general population (40) or to quickly recruit subjects on which verify individual's personal experiences and sociocultural factors (41). In addition to increasing awareness of HPV vaccines through health education, communication on the risks of HPV vaccines can help build public confidence and trust. The vaccine management party or the health department can set up a risk communication department to conduct preventive risk communication. Avoid increasing vaccine hesitancy due to asymmetric risk information.

Limitations of this study include that there was no segmentation of vaccine hesitancy measurements and that we gathered information only on whether vaccine hesitancy occurred but not on the frequency and degree of vaccine hesitancy. The measurement of the degree is a very complicated issue. Even if there are HPV vaccine hesitancy scales developed in other countries, the applicability of the scales in China has yet to be verified. Using Likert scale and other tools to measure the degree of hesitancy will be our next research content. The study included 728 participants, and the study may have representative questions. However, the number of medical students in China is limited, and we have distributed the samples to different regions of China as much as possible. Another limitation was the cross-sectional design, which cannot provide causal relationships. Despite these limitations, the survey gained a high response rate, showing a strong interest in the HPV topic.

\section{CONCLUSIONS}

Vaccine hesitancy is prevalent among Chinese medical students. Medical students are potential beneficiaries of the HPV vaccine and a key force for future vaccination; thus, it is necessary to implement measures that address the reasons for their reported vaccine hesitancy. Making vaccination convenient by the use of mobile vaccination vehicles and increasing vaccination sites such as in schools or pharmacies could be an effective way to reduce HPV vaccine hesitancy. The role of medical workers in expanding respondents' understanding of the safety and effectiveness of the vaccine may also help reduce vaccine hesitancy. Additionally, the role of online media in vaccine hesitancy should also be fully considered.

\section{DATA AVAILABILITY STATEMENT}

The raw data supporting the conclusions of this article will be made available by the authors, without undue reservation.

\section{ETHICS STATEMENT}

The study was conducted according to the guidelines of the Declaration of Helsinki, and approved by the Harbin Medical University School of Health Management and Institutional Research Board (reference no. HMUIRB20210006, approved on 
30 June 2021). Written informed consent for participation was not required for this study in accordance with the national legislation and the institutional requirements.

\section{AUTHOR CONTRIBUTIONS}

LZ, JW, YL, PC, XZ, and GL: conceptualization. LZ, JW, YL, and PC: methodology, data curation, and formal analysis. LZ, XZ, and GL: validation. YL, PC, JW, and XZ: investigation. $\mathrm{XZ}$ and GL: resources, supervision, and project

\section{REFERENCES}

1. World Health Organization. Human Papillomavirus (HPV) and Cervical Cancer (2020). Available online at: https://www.who.int/zh/news-room/factsheets/detail/human-papillomavirus-(hpv)-and-cervical-cancer (accessed on June 15, 2021).

2. World Health Organization. Human Papillomavirus Vaccines. WHO Position Paper. J Biol Stand. (2009) 37:338-44. doi: 10.1016/j.biologicals.2009.04.005

3. World Health Organization. Electronic address: sageexecsec@who.int. Human papillomavirus vaccines: WHO position paper, May 2017-Recommendations. Vaccine. (2017) 35:57535. doi: 10.1016/j.vaccine.2017.05.069

4. World Health Organization. Cervical Cancer (2021). Available online at: https://www.who.int/health-topics/cervical-cancer\%23tab=tab_1 (accessed June 15, 2021).

5. Sung H, Ferlay J, Siegel RL, Laversanne M, Soerjomataram I, Jemal A, et al. Global cancer statistics 2020: GLOBOCAN estimates of incidence and mortality worldwide for 36 cancers in 185 countries. CA Cancer J Clin. (2021) 71:209-49. doi: 10.3322/caac. 21660

6. Mattoscio D, Medda A, Chiocca S. Human papilloma virus and autophagy. Int J Mol Sci. (2018) 19:1775. doi: 10.3390/ijms19061775

7. Drolet M, Bénard É, Pérez N, Brisson M, HPV Vaccination Impact Study Group. Population-level impact and herd effects following the introduction of human papillomavirus vaccination programmes: updated systematic review and meta-analysis. Lancet. (2019) 394:497-509. doi: 10.1016/S0140-6736(19)30298-3

8. Bianchi FP, Gallone MS, Fortunato F, Boccalini S, Martinelli D, Prato R, et al. Epidemiology and cost of cervical cancer care and prevention in Apulia (Italy), 2007/2016. Ann Ig. (2018) 30:490-501. doi: 10.7416/ai.2018.2249

9. World Health Organization. Immunization. Available online at: https://www. who.int/topics/immunization/zh/ (accessed June 15, 2021).

10. Thomas TL. Cancer Prevention: HPV vaccination. Semin Oncol Nurs. (2016) 32:273-80. doi: 10.1016/j.soncn.2016.05.007

11. Jit M, Brisson M, Portnoy A, Hutubessy R. Cost-effectiveness of female human papillomavirus vaccination in 179 countries: a PRIME modelling study. Lancet Glob Health. (2014) 2:e406-14. doi: 10.1016/S2214-109X(14)70237-2

12. Mo X, Gai Tobe R, Wang L, Liu X, Wu B, Luo H, et al. Cost-effectiveness analysis of different types of human papillomavirus vaccination combined with a cervical cancer screening program in mainland China. BMC Infect Dis. (2017) 17:502. doi: 10.1186/s12879-017-2592-5

13. Van Minh H, My NTT, Jit M. Cervical cancer treatment costs and cost-effectiveness analysis of human papillomavirus vaccination in Vietnam: a PRIME modeling study. BMC Health Serv Res. (2017) 17:353. doi: 10.1186/s12913-017-2297-x

14. Chesson HW, Meites E, Ekwueme DU, Saraiya M, Markowitz LE. Updated medical care cost estimates for HPV-associated cancers: implications for costeffectiveness analyses of HPV vaccination in the United States. Hum Vaccin Immunother. (2019) 15:1942-8. doi: 10.1080/21645515.2019.1603562

15. World Health Organization. Ten Threats to Global Health in 2019 (2019). Available online at: https://www.who.int/emergencies/ten-threats-to-globalhealth-in-2019 (accessed June 19, 2021). administration. LZ: writing-original draft preparation. XZ: writing-review and editing. YL, PC, and JW: visualization. All authors contributed to the article and approved the submitted version.

\section{SUPPLEMENTARY MATERIAL}

The Supplementary Material for this article can be found online at: https://www.frontiersin.org/articles/10.3389/fpubh. 2022.774767/full\#supplementary-material

16. MacDonald NE, SAGE Working Group on Vaccine Hesitancy. Vaccine hesitancy: definition, scope and determinants. Vaccine. (2015) 33:41614. doi: 10.1016/j.vaccine.2015.04.036

17. Hanley SJ, Yoshioka E, Ito Y, Kishi R. HPV vaccination crisis in Japan. Lancet. (2015) 385:2571. doi: 10.1016/S0140-6736(15)61152-7

18. Costa AS, Gomes JM, Germani ACCG, da Silva MR, Santos EFS, Soares Júnior JM, et al. Knowledge gaps and acquisition about HPV and its vaccine among Brazilian medical students. PLoS ONE. (2020) 15:e0230058. doi: 10.1371/journal.pone.0230058

19. Wang X, Du T, Shi X, Wu K. Awareness and knowledge about human papilloma virus infection among students at secondary occupational health school in China. Int J Environ Res Public Health. (2021) 18:6321. doi: 10.3390/ijerph18126321

20. Lee PW, Kwan TT, Tam KF, Chan KK, Young PM, Lo SS, et al. Beliefs about cervical cancer and human papillomavirus (HPV) and acceptability of HPV vaccination among Chinese women in Hong Kong. Prev Med. (2007) 45:130-4. doi: 10.1016/j.ypmed.2007.07.013

21. World Health Organization. Appendices to the Report of the Sage Working Group on Vaccine Hesitancy (2014). Available online at: https://www.who. int/immunization/sage//meetings/2014/october/2_SAGE_Appendicies_ Background_final.pdf?ua=1 (accessed June 20, 2021).

22. Du F, Chantler T, Francis MR, Sun FY, Zhang X, Han K, et al. Access to Vaccination information and confidence/hesitancy towards childhood vaccination: a cross-sectional survey in China. Vaccines. (2021) 9:201. doi: 10.3390/vaccines9030201

23. China National Medical Products Administration. HPV Vaccine and Prevention of Cervical Cancer [in Chinese] (2019). Available online at: https://www.nmpa.gov.cn/yaopin/ypjgdt/20190722080001877.html (accessed June 21, 2021).

24. Matsuo K, Mabuchi S, Okazawa M, Kawano M, Kuroda H, Kamiura $\mathrm{S}$, et al. Clinical implication of surgically treated early-stage cervical cancer with multiple high-risk factors. J Gynecol Oncol. (2015) 26:311. doi: 10.3802/jgo.2015.26.1.3

25. Fu CJ, Pan XF, Zhao ZM, Saheb-Kashaf M, Chen F, Wen Y, et al. Knowledge, perceptions and acceptability of HPV vaccination among medical students in Chongqing, China. Asian Pac J Cancer Prev. (2014) 15:618793. doi: 10.7314/apjcp.2014.15.15.6187

26. Schmitt HJ, Booy R, Aston R, Van Damme P, Schumacher RF, Campins $\mathrm{M}$, et al. How to optimise the coverage rate of infant and adult immunisations in Europe. BMC Med. (2007) 5:11. doi: 10.1186/1741-70 15-5-11

27. Santhanes D, Yong CP, Yap YY, Saw PS, Chaiyakunapruk N, Khan TM. Factors influencing intention to obtain the HPV vaccine in South East Asian and Western Pacific regions: a systematic review and meta-analysis. Sci Rep. (2018) 8:3640. doi: 10.1038/s41598-018-21912-x

28. US Centers for Disease Control and Prevention. Safety Information for HPV Vaccine (2020). Available online at: https://www.cdc.gov/vaccinesafety/ vaccines/hpv-vaccine.html (accessed June 23, 2021).

29. Karafillakis E, Dinca I, Apfel F, Cecconi S, Wurz A, Takacs J, et al. Vaccine hesitancy among healthcare workers in Europe: a qualitative study. Vaccine. (2016) 34:5013-20. doi: 10.1016/j.vaccine.2016.08.029 
30. Taddio A, Appleton M, Bortolussi R, Chambers C, Dubey V, Halperin S, et al. Reducing the pain of childhood vaccination: an evidence-based clinical practice guideline. CMAJ. (2010) 182:E843-55. doi: 10.1503/cmaj.101720

31. Icardi G, Costantino C, Guido M, Zizza A, Restivo V, Amicizia D, et al. Burden and Prevention of HPV. Knowledge, practices and attitude assessment among pre-adolescents and their parents in Italy. Curr Pharm Des. (2020) 26:326-42. doi: 10.2174/1381612826666200114100553

32. Malo TL, Ali KN, Sutton SK, Perkins RB, Giuliano AR, Vadaparampil ST. The content and context of physicians' communication with males about human papillomavirus vaccination. Hum Vaccin Immunother. (2016) 12:1511-8. doi: 10.1080/21645515.2015.1132963

33. Newcomer SR, Caringi J, Jones B, Coyle E, Schehl T, Daley MF. A mixedmethods analysis of barriers to and facilitators of human papillomavirus vaccination among adolescents in Montana. Public Health Rep. (2020) 135:842-50. doi: 10.1177/0033354920954512

34. Fokoun C. Strategies implemented to address vaccine hesitancy in France: a review article. Hum Vaccin Immunother. (2018) 14:158090. doi: 10.1080/21645515.2018.1458807

35. Stahl JP, Cohen R, Denis F, Gaudelus J, Martinot A, Lery T, et al. The impact of the web and social networks on vaccination. New challenges and opportunities offered to fight against vaccine hesitancy. Med Mal Infect. (2016) 46:117-22. doi: 10.1016/j.medmal.2016.02.002

36. Piedrahita-Valdés H, Piedrahita-Castillo D, Bermejo-Higuera J, Guillem-Saiz P, Bermejo-Higuera JR, Guillem-Saiz J, et al. Vaccine hesitancy on social media: sentiment analysis from June 2011 to April 2019. Vaccines. (2021) 9:28. doi: 10.3390/vaccines9010028

37. Lucia VC, Kelekar A, Afonso NM. COVID-19 vaccine hesitancy among medical students. J Public Health. (2020) 43(3):4459. doi: 10.1093/pubmed/fdaa230

38. Griffith J, Marani H, Monkman H. COVID-19 vaccine hesitancy in Canada: content analysis of tweets using the theoretical domains framework. J Med Internet Res. (2021) 23:e26874. doi: 10.2196/26874
39. Bratic JS, Seyferth ER, Bocchini JA Jr. Update on barriers to human papillomavirus vaccination and effective strategies to promote vaccine acceptance. Curr Opin Pediatr. (2016) 28:407-12. doi: 10.1097/MOP.0000000000000353

40. Zareba K, La Rosa VL, Kołb-Sielecka E, Ciebiera M, Ragusa R, Gierus J, et al. Attitudes and opinions of young gynecologists on pregnancy termination: results of a cross-sectional survey in Poland. Int J Environ Res Public Health. (2020) 17:3895. doi: 10.3390/ijerph17113895

41. Rodrigues Eberhardt P, La Rosa VL, Marques Cerentini T, Caruso S, Ragusa R, De Franciscis P, et al. Sexual dysfunctions in female university students and the correlation with body image: a cross-sectional study. J Psychosom Obstet Gynaecol. (2020) 19:1-6. doi: 10.1080/0167482X.2020. 1806818

Conflict of Interest: The authors declare that the research was conducted in the absence of any commercial or financial relationships that could be construed as a potential conflict of interest.

Publisher's Note: All claims expressed in this article are solely those of the authors and do not necessarily represent those of their affiliated organizations, or those of the publisher, the editors and the reviewers. Any product that may be evaluated in this article, or claim that may be made by its manufacturer, is not guaranteed or endorsed by the publisher.

Copyright (C) 2022 Zhou, Wang, Cheng, Li, Liu and Zhang. This is an open-access article distributed under the terms of the Creative Commons Attribution License (CC $B Y)$. The use, distribution or reproduction in other forums is permitted, provided the original author(s) and the copyright owner(s) are credited and that the original publication in this journal is cited, in accordance with accepted academic practice. No use, distribution or reproduction is permitted which does not comply with these terms. 